\title{
ERRATUM
}

\section{Dynamic Load Balancing on Dedicated Heterogeneous Systems}

\author{
Ismael Galindo $^{1}$, Francisco Almeida ${ }^{1}$, and José Manuel Badía-Contelles ${ }^{2}$ \\ ${ }^{1}$ Department of Statistics and Computer Science \\ La Laguna University Spain \\ ${ }^{2}$ Department of Computer Science and Engineering \\ Jaume I University Spain
}

\begin{abstract}
A. Lastovetsky et al. (Eds.): EuroPVM/MPI 2008, LNCS 5205, pp. 64-74, 2008.
(C) Springer-Verlag Berlin Heidelberg 2008
\end{abstract}

DOI 10.1007/978-3-540-87475-1_50

In the original version, the author name Vicente Blanco has been inadvertently missed out. The affiliation is Department of Statistics and Computer Science, La Laguna University, Spain. The correct running head for this chapter is I. Galindo et al. 